\title{
A fast collocation method for a variable-coefficient nonlocal diffusion model
}

\author{
Che Wang Hong Wang \\ Department of Mathematics, University of South Carolina, \\ Columbia, South Carolina 29208, USA (e-mail address: hwang@math.sc.edu, telephone: \\ 803-777-4321. fax: 803-777-6527)
}

\begin{abstract}
We develop a fast collocation scheme for a variable-coefficient nonlocal diffusion model, for which a numerical discretization would yield a dense stiffness matrix. The development of the fast method is achieved by carefully handling the variable coefficients appearing inside the singular integral operator and exploiting the structure of the dense stiffness matrix. The resulting fast method reduces the computational work from $O\left(N^{3}\right)$ required by a commonly used direct solver to $O(N \log N)$ per iteration and the memory requirement from $O\left(N^{2}\right)$ to $O(N)$. Furthermore, the fast method reduces the computational work of assembling the stiffness matrix from $O\left(N^{2}\right)$ to $O(N)$. Numerical results are presented to show the utility of the fast method.
\end{abstract}

Keywords: fast collocation method, fractional diffusion equation, nonlocal diffusion model, nonlocal model

\section{Introduction}

Nonlocal models provide an alternative means to fractional partial differential equations $[2,9,13,16,17]$ in modeling challenging phenomena such as anomalous diffusion and long-range spatial interactions, which cannot be modeled properly by conventional integer-order differential equations $[7,8,11]$. However, its numerical discretizations yield dense stiffness matrices, for which commonly used direct solvers require $O\left(N^{2}\right)$ memory storage and $O\left(N^{3}\right)$ computational cost where $N$ is the number of unknowns. These significantly increased computational complexity and memory requirement becomes one of the main obstacles that hinders its applications. 
Motivated by our development of fast numerical methods for fractional differential equations $[1,22,26]$ and their relationship to nonlocal diffusion models, we developed fast numericnal methods for a one-dimensional peridynamic model [21, 24] and a two-dimensional nonlocal diffusion method [25]. This was done by fully utilizing the Toeplitz-like structure of the stiffness matrix, which reduces the computational complexity from $O\left(N^{3}\right)$ to $O(N \log N)$ and memory requirement from $O\left(N^{2}\right)$ to $O(N)$. Numerical experiments show the utility of the fast methods.

Recently, Mengesha and Du [15] developed a variable-coefficient peridynamic model to account for the heterogeneity of the elastic material, to which the fast numerical methods developed in [24, 25] do not apply. Moreover, the variable coefficents in the space-fractional differential equation (refer to (9)) appear in front of the fractional derivatives, leading to diagonal matrix multipliers in the Toeplitz-like matrix decomposition of the stiffness matrix (refer to (14)).

However, for a variable-coefficient nonlocal diffusion model (refer to (1)), the variable cofficients occur inside the integral operator. This would bring a global impact of the variable coefficients to its numerical discretization, in which the number of summands in the matrix decomposition in [24, 26] would be on the order of $O(N)$. Consequently, the corresponding matrix-vector multiplication would be on the order of $O\left(N^{2}\right)$. In this paper we develop an alternative way to handle the nonlocal impact of the variable coefficients in the discretization of the nonlocal diffusion model, so that the resulting numerical method has an optimal-order memory storage and an approximately linear computational complexity. The rest of the paper is organized as follows. In $\S 2$ we present a nonlocal diffusion model to be considered in this paper and a corresponding collocation scheme. In $\S 3$ we demonstrate the different features of the numerical methods for a nonlocal diffusion model and a space-fractional partial differential equation. In $\S 4$ we study an effective and efficient evaluation of the stiffness matrix. In $\S 5$ we study the structure of the stiffness matrix and prove a Toeplitz-like decomposition for the stiffness matrix, which can then be applied to develop any Krylov subspace iterative solvers. In $\S 6$ we conduct numerical experiments to investigate the computational benefits of the fast method developed. 


\section{A nonlocal diffusion model and its collocation discretization}

A volume constrained nonlocal Dirichlet boundary-value problem of a variable-coefficient nonlocal diffusion model may be formulated as follows $[7,8,11,15]$

$$
\begin{gathered}
\int_{x-\delta}^{x+\delta}(\alpha(x)+\alpha(y)) \sigma(x-y)(u(x)-u(y)) d y=f(x), \quad x \in(a, b) \\
u(x)=g(x), \quad x \in(a-\delta, a] \cup[b, b+\delta) .
\end{gathered}
$$

Here $\delta>0$ refers to the size of the horizon which specifies the range of the nonlocal diffusion phenomenon, and $\alpha(\cdot)$ is the variable diffusivity coefficient which is assumed to have positive lower and upper bounds and to be smooth. The kernel $\sigma(x)$ is defined by

$$
\sigma(x):=\frac{1}{|x|^{1+\gamma}}
$$

The index $\gamma<1$ specifies the intensity of the kernel, so the integral operator is weakly singular.

Let $N$ be a positive integer and the mesh size $h:=(b-a) / N$. We define $K$ to the floor of $\delta / h$

$$
K:=\lfloor\delta / h\rfloor .
$$

$\{\mathrm{Col}: \mathrm{e} 0\}$

We define a uniform partition on the interval $(a-\delta, b+\delta)$ by $x_{i}:=a+i h$ for $-K \leq i \leq N+K$. Let $\psi(\xi):=1-|\xi|$ for $\xi \in[-1,1]$ and 0 elsewhere. Then we let $\phi_{i}(x):=\psi\left(\left(x-x_{i}\right) / h\right)$ be the hat functions centered at $x=x_{i}$ for $i=0,1, \ldots, N$. The trial function $u$ can be chosen as

$$
u(x)=\sum_{j=-K}^{N+K} u_{j} \phi_{j}(x)=\sum_{j=1}^{N-1} u_{j} \phi_{j}(x)+\left(\sum_{j=0}^{-K}+\sum_{j=N}^{N+K}\right) g\left(x_{j}\right) \phi_{j}(x) .
$$

We substitute the trial function (4) into (5) and enforce the governing equation in (1) at the collocation points $\left\{x_{i}\right\}_{i=1}^{N-1}$ to obtain

$$
\begin{gathered}
\int_{x_{i}-\delta}^{x_{i}+\delta} \frac{\left(\alpha\left(x_{i}\right)+\alpha(y)\right)}{\left|x_{i}-y\right|^{1+\gamma}}\left(u\left(x_{i}\right)-\sum_{j=-K}^{N+K} u_{j} \phi_{j}(y)\right) d y=f\left(x_{i}\right), \\
1 \leq i \leq N-1
\end{gathered}
$$


We express these equations in terms of the nodal values $u_{j}$ as follows

$$
\begin{aligned}
& u_{i} \int_{x_{i}-\delta}^{x_{i}+\delta} \frac{\left(\alpha\left(x_{i}\right)+\alpha(y)\right)\left(1-\phi_{i}(y)\right)}{\left|x_{i}-y\right|^{1+\gamma}} d y \\
& \quad-\sum_{j=1, j \neq i}^{N-1} u_{j} \int_{x_{i}-\delta}^{x_{i}+\delta} \frac{\left(\alpha\left(x_{i}\right)+\alpha(y)\right) \phi_{j}(y)}{\left|x_{i}-y\right|^{1+\gamma}} d y \\
& =f\left(x_{i}\right)+\left(\sum_{j=0}^{-K}+\sum_{j=N}^{N+K}\right) g\left(x_{j}\right) \int_{x_{i}-\delta}^{x_{i}+\delta} \frac{\left(\alpha\left(x_{i}\right)+\alpha(y)\right) \phi_{j}(y)}{\left|x_{i}-y\right|^{1+\gamma}} d y \\
& 1 \leq i \leq N-1 .
\end{aligned}
$$

The numerical scheme (6) can be formulated in a matrix form

$$
\mathrm{Au}=\mathbf{f}
$$

Here the unknown vector $\mathbf{u}:=\left[u_{1}, u_{2}, \ldots, u_{N-1}\right]^{T}$ with $\left\{u_{j}\right\}_{j=1}^{N-1}$ being given in (4), and the stiffness matrix $\mathbf{A}:=\left[A_{i, j}\right]_{i, j=1}^{N-1}$ and the right-hand side $\mathbf{f}:=$ $\left[f_{1}, f_{2}, \ldots, f_{N-1}\right]^{T}$ are defined by

$$
\begin{aligned}
A_{i, j}= & \int_{x_{i}-\delta}^{x_{i}+\delta} \frac{\left(\alpha\left(x_{i}\right)+\alpha(y)\right) \phi_{j}(y)}{\left|x_{i}-y\right|^{1+\gamma}} d y, \quad j \neq i \\
A_{i, i}= & \int_{x_{i}-\delta}^{x_{i}+\delta} \frac{\left(\alpha\left(x_{i}\right)+\alpha(y)\right)\left(1-\phi_{i}(y)\right)}{\left|x_{i}-y\right|^{1+\gamma}} d y \\
f_{i}= & \left\{\begin{array}{c}
f\left(x_{i}\right)+\sum_{j=0}^{-K} g\left(x_{j}\right) \int_{x_{i}-\delta}^{x_{i}+\delta} \frac{\left(\alpha\left(x_{i}\right)+\alpha(y)\right) \phi_{j}(y)}{\left|x_{i}-y\right|^{1+\gamma}} d y \\
1 \leq i \leq K+1, \\
f\left(x_{i}\right), \quad K+2 \leq i \leq N-K-2, \\
f\left(x_{i}\right)+\sum_{j=N}^{N+K} g\left(x_{j}\right) \int_{x_{i}-\delta}^{x_{i}+\delta} \frac{\left(\alpha\left(x_{i}\right)+\alpha(y)\right) \phi_{j}(y)}{\left|x_{i}-y\right|^{1+\gamma}} d y, \\
N-K-1 \leq i \leq N-1 .
\end{array}\right.
\end{aligned}
$$

We observe from (8) that each row of the stiffness matrix $\mathbf{A}$ has $O(K)$ nonzero entries. We note that $K=O(N)$ as $N$ increases. In other words, the stiffness matrix $\mathbf{A}$ is asymptotically a dense matrix, for which a direct solver would require $O\left(N^{2}\right)$ storage and has $O\left(N^{3}\right)$ computational complexity. 


\section{Features of numerical methods for a nonlocal diffusion model and a space-fractional diffusion equation}

We note that the nonlocal diffusion model (1) is closely related to a spacefractional differential equation [8]. Our previous development of fast finite difference methods for space-fractional diffusion equations [26, 27] motivates the current development of a fast collocation method for the nonlocal diffusion model (1). Recall that a space-fractional diffusion equation of order $1<\alpha<2$ can be formulated as follows $[9,14]$

$$
\begin{aligned}
& u_{t}-d_{+}(x, t)_{a}^{l} D_{x}^{\alpha} u-d_{-}(x, t)_{x}^{r} D_{b}^{\alpha} u=f(x, t), \quad(x, t) \in(a, b) \times(0, T], \\
& u(x, 0)=u_{0}(x), x \in[a, b], \quad u(a, t)=u(b, t)=0, t \in[0, T] .
\end{aligned}
$$

Here $d_{+}$and $d_{-}$are the left and right diffusivity coefficients, and $f$ is the source term.

Let $t^{m}:=m \tau$ for $0 \leq m \leq M$ with $\tau:=T / M$. The implicit finite difference method for problem (9) is of the form [14]

$$
\begin{gathered}
\frac{u_{i}^{m}-u_{i}^{m-1}}{\tau}-\frac{d_{i}^{+, m}}{h^{\alpha}} \sum_{k=0}^{i} g_{k}^{(\alpha)} u_{i-k+1}^{m}-\frac{d_{i}^{-, m}}{h^{\alpha}} \sum_{k=0}^{N-i} g_{k}^{(\alpha)} u_{i+k-1}^{m}=f_{i}^{m}, \\
1 \leq i \leq N-1, \quad 1 \leq m \leq M
\end{gathered}
$$

with $d_{i}^{ \pm, m}:=d_{ \pm}\left(x_{i}, t^{m}\right), f_{i}^{m}:=f\left(x_{i}, t^{m}\right)$, and $u_{i}^{m}$ the numerical solution at $\left(x_{i}, t^{m}\right) \cdot g_{k}^{(\alpha)}=(-1)^{k}\left(\begin{array}{l}\alpha \\ k\end{array}\right)$ with $\left(\begin{array}{l}\alpha \\ k\end{array}\right)$ being the fractional binomial coefficients. The finite difference scheme (10) can be expressed in a matrix form

$$
\left(\mathbf{I}+\tau \mathbf{A}^{m}\right) \mathbf{u}^{m}=\mathbf{u}^{m-1}+\tau \mathbf{f}^{m}, \quad 1 \leq m \leq M .
$$

Here $\mathbf{u}^{m}:=\left[u_{1}^{m}, \ldots, u_{N-1}^{m}\right]^{T}, \mathbf{f}^{m}:=\left[f_{1}^{m}, \ldots, f_{N-1}^{m}\right]^{T}$, and $\mathbf{I}$ is the identity matrix of order $N-1$, and the matrices $\mathbf{A}^{m}=\left[a_{i, j}^{m}\right]_{i, j=1}^{N-1}$ are defined by

$$
a_{i, j}^{m}:= \begin{cases}-\left(r_{i}^{+, m}+r_{i}^{-, m}\right) g_{1}^{(\alpha)}, & j=i, \\ -r_{i}^{+, m} g_{2}^{(\alpha)}-r_{i}^{-, m} g_{0}^{(\alpha)}, & j=i-1, \\ -r_{i}^{+, m} g_{0}^{(\alpha)}-r_{i}^{-, m} g_{2}^{(\alpha)}, & j=i+1, \\ -r_{i}^{+, m} g_{i-j+1}^{(\alpha)}, & j<i-1, \\ -r_{i}^{-, m} g_{j-i+1}^{(\alpha)}, & j>i+1\end{cases}
$$


where

$$
r_{i}^{+, m}:=\frac{d_{i}^{+, m} \tau}{h^{\alpha}}, \quad r_{i}^{-, m}:=\frac{d_{i}^{-, m} \tau}{h^{\alpha}},
$$

$\{$ NFDE: e5 $\}$

and $g^{\alpha}$ is the fractional binomial coefficient.

We proved in [26] that the stiffness matrix $\mathbf{A}^{m}$ of the finite difference scheme (10) can be decomposed as

$$
\mathbf{A}^{m}=\operatorname{diag}\left(\mathbf{r}_{+}^{m}\right) \mathbf{T}^{\alpha, N-1}+\operatorname{diag}\left(\mathbf{r}_{-}^{m}\right)\left(\mathbf{T}^{\alpha, N-1}\right)^{T} .
$$

Here $\mathbf{r}_{ \pm}^{m}=\left[r_{ \pm, 1}^{m}, \ldots, r_{ \pm, N-1}^{m}\right]^{T}$ with $r_{ \pm, i}^{m}$ defined in (13), $\mathbf{T}^{\alpha, N-1}$ is a lower Hessenberg Toeplitz matrix of order $N-1$ defined by

$$
\mathbf{T}^{\alpha, N-1}:=\left[\begin{array}{cccccc}
g_{1}^{(\alpha)} & g_{0}^{(\alpha)} & 0 & \ldots & 0 & 0 \\
g_{2}^{(\alpha)} & g_{1}^{(\alpha)} & g_{0}^{(\alpha)} & \ddots & \ddots & 0 \\
\vdots & g_{2}^{(\alpha)} & g_{1}^{(\alpha)} & \ddots & \ddots & \vdots \\
\vdots & \ddots & \ddots & \ddots & \ddots & 0 \\
g_{N-2}^{(\alpha)} & \ddots & \ddots & \ddots & g_{1}^{(\alpha)} & g_{0}^{(\alpha)} \\
g_{N-1}^{(\alpha)} & g_{N-2}^{(\alpha)} & \ldots & \ldots & g_{2}^{(\alpha)} & g_{1}^{(\alpha)}
\end{array}\right]
$$

Fast numerical methods were developed in $[26,27]$ based on the decomposition (14).

In this paper we develop a fast collocation method for the nonlocal diffusion model (1) by prove a decomposition similar to (14). However, two major differences occur in the current context:

- The evaluation of stiffness matrix $\mathbf{A}^{m}$ in the finite difference scheme (10) is straightforward. However, the evaluation of the stiffness matrix A in the collocation method (7)-(8) requires the evaluation of singular integrals in all the nonzero entries of $\mathbf{A}$.

- The variable coefficients in the space-fractional differential equation (9) appear in front of the fractional derivatives, leading to diagonal matrices in the decomposition (14). However, in the context of the nonlocal diffusion model (1), the variable coefficients occur inside the integral operator that introduce nonlocal influence of a range $\left(x_{i}-\right.$ $\left.\delta, x_{i}+\delta\right)$ at each node $x_{i}$ for $i=1,2, \ldots, N-1$. 


\section{Evaluation of the stiffness matrix}

We shall study an efficient evaluation of the stiffness matrix A. Recall that the stiffness matrices of numerical methods for integer-order partial differential equations are sparse. Consequently, the evaluation of these matrices naturally has linear complexity. However, for the nonlocal diffusion model (1), the stiffness matrix $\mathbf{A}$ is dense that requires $O\left(N^{2}\right)$ computational work to assemble and $O\left(N^{2}\right)$ memory to store. For a constant-coefficient analogue of the nonlocal diffusion model (1), we proved a Toeplitz-like structure of the stiffness matrix of the corresponding numerical methods [24, 25]. However, the presence of a variable coefficient in the model (1) destroys the Toeplitzlike structure of the stiffness matrix $\mathbf{A}$ of the numerical scheme (7)-(8). To develop a fast method, we first evaluate the stiffness matrix $\mathbf{A}$.

\subsection{Evaluation of the diagonal entries of the stiffness matrix}

We begin by evaluating the diagonal entries of the stiffness matrix $A$ in (8) for $i=1,2, \ldots, N-1$

$$
A_{i, i}=\alpha\left(x_{i}\right) \int_{x_{i}-\delta}^{x_{i}+\delta} \frac{1-\phi_{i}(y)}{\left|x_{i}-y\right|^{1+\gamma}} d y+\int_{x_{i}-\delta}^{x_{i}+\delta} \frac{\alpha(y)\left(1-\phi_{i}(y)\right)}{\left|x_{i}-y\right|^{1+\gamma}} d y
$$

We utilize the symmetry of the integrand to evaluate the first term on the right-hand as follows

$$
\begin{aligned}
& \int_{x_{i}-\delta}^{x_{i}+\delta} \frac{1-\phi_{i}(y)}{\left|x_{i}-y\right|^{1+\gamma}} d y \\
& \quad=2 \int_{x_{i+1}}^{x_{i}+\delta} \frac{1}{\left(y-x_{i}\right)^{1+\gamma}} d y+2 \int_{x_{i}}^{x_{i+1}} \frac{1-\phi_{i}(y)}{\left(y-x_{i}\right)^{1+\gamma}} d y \\
& \quad=\frac{2\left(h^{-\gamma}-\delta^{-\gamma}\right)}{\gamma}+\frac{2 h^{-\gamma}}{1-\gamma} .
\end{aligned}
$$

However, the evaluation of the second integral on the right-hand side of (16) is much more involved, as pointed out earlier. As the variable-coefficient $\alpha(y)$ is inside the integration with respect to $y$, a numerical quadrature has to be applied to evaluate the integral in general. Furthermore, the domain of integration, i.e., the horizon of the material at the collocation point $x_{i}$, is $\left(x_{i}-\delta, x_{i}+\delta\right)$, which is asymptotically of order $O(N)$. A naive application of a numerical quadrature would require $O\left(N^{2}\right)$ computations to evaluate all 
the diagonal entries of the matrix $\mathbf{A}$. To reduce the computational work, we decompose this term as

$$
\begin{aligned}
\int_{x_{i}-\delta}^{x_{i}+\delta} & \frac{\alpha(y)\left(1-\phi_{i}(y)\right)}{\left|x_{i}-y\right|^{1+\gamma}} d y \\
= & \int_{x_{i}-\delta}^{x_{i-1}} \frac{\alpha(y)}{\left(x_{i}-y\right)^{1+\gamma}} d y+\int_{x_{i+1}}^{x_{i}+\delta} \frac{\alpha(y)}{\left(y-x_{i}\right)^{1+\gamma}} d y \\
& +\int_{x_{i-1}}^{x_{i}} \frac{\alpha(y)\left(1-\phi_{i}(y)\right)}{\left(x_{i}-y\right)^{1+\gamma}} d y+\int_{x_{i}}^{x_{i+1}} \frac{\alpha(y)\left(1-\phi_{i}(y)\right)}{\left(y-x_{i}\right)^{1+\gamma}} d y .
\end{aligned}
$$

$\{$ Mati:e2a\}

We evaluate the fourth term as

$$
\begin{aligned}
\int_{x_{i}}^{x_{i+1}} & \frac{\alpha(y)\left(1-\phi_{i}(y)\right)}{\left(y-x_{i}\right)^{1+\gamma}} d y=\frac{1}{h} \int_{x_{i}}^{x_{i+1}} \frac{\alpha(y)}{\left(y-x_{i}\right)^{\gamma}} d y \\
= & \frac{1}{h} \int_{x_{i}}^{x_{i+1}} \frac{\alpha(y)-\alpha\left(x_{i}\right)}{\left(y-x_{i}\right)^{\gamma}} d y+\frac{\alpha\left(x_{i}\right)}{h} \int_{x_{i}}^{x_{i+1}} \frac{1}{\left(y-x_{i}\right)^{\gamma}} d y \\
\approx & \frac{1}{h} \int_{x_{i}}^{x_{i+1}} \frac{\alpha^{\prime}\left(x_{i}\right)\left(y-x_{i}\right)+\frac{\alpha^{\prime \prime}\left(x_{i}\right)\left(y-x_{i}\right)^{2}}{2}+\frac{\alpha^{\prime \prime \prime}\left(x_{i}\right)\left(y-x_{i}\right)^{3}}{6}}{\left(y-x_{i}\right)^{\gamma}} d y \\
& +\frac{\alpha\left(x_{i}\right)}{h} \int_{x_{i}}^{x_{i+1}} \frac{1}{\left(y-x_{i}\right)^{\gamma}} d y \\
= & \frac{\alpha\left(x_{i}\right) h^{-\gamma}}{1-\gamma}+\frac{\alpha^{\prime}\left(x_{i}\right) h^{1-\gamma}}{2-\gamma}+\frac{\alpha^{\prime \prime}\left(x_{i}\right) h^{2-\gamma}}{2(3-\gamma)}+\frac{\alpha^{\prime \prime \prime}\left(x_{i}\right) h^{3-\gamma}}{6(4-\gamma)} .
\end{aligned}
$$

We similarly use the Taylor expansion to evaluate the second term on the right-hand side of (18) as follows

$$
\begin{aligned}
\int_{x_{i+1}}^{x_{i}+\delta} \frac{\alpha(y)}{\left(y-x_{i}\right)^{1+\gamma}} d y & =\frac{\alpha\left(x_{i}\right)\left(h^{-\gamma}-\delta^{-\gamma}\right)}{\gamma}+\frac{\alpha^{\prime}\left(x_{i}\right)\left(\delta^{1-\gamma}-h^{1-\gamma}\right)}{1-\gamma} \\
& +\frac{\alpha^{\prime \prime}\left(x_{i}\right)\left(\delta^{2-\gamma}-h^{2-\gamma}\right)}{2(2-\gamma)}+\frac{\alpha^{\prime \prime \prime}\left(x_{i}\right)\left(\delta^{3-\gamma}-h^{3-\gamma}\right)}{6(3-\gamma)}
\end{aligned}
$$

By symmetry, we evaluate the first and third terms on the right-hand side of (18) as

$$
\begin{aligned}
\int_{x_{i-\delta}}^{x_{i-1}} \frac{\alpha(y)}{\left(x_{i}-y\right)^{1+\gamma}} d y & \approx \frac{\alpha\left(x_{i}\right)\left(h^{-\gamma}-\delta^{-\gamma}\right)}{\gamma}-\frac{\alpha^{\prime}\left(x_{i}\right)\left(\delta^{1-\gamma}-h^{1-\gamma}\right)}{1-\gamma} \\
& +\frac{\alpha^{\prime \prime}\left(x_{i}\right)\left(\delta^{2-\gamma}-h^{2-\gamma}\right)}{2(2-\gamma)}-\frac{\alpha^{\prime \prime \prime}\left(x_{i}\right)\left(\delta^{3-\gamma}-h^{3-\gamma}\right)}{6(3-\gamma)}
\end{aligned}
$$


and

$$
\begin{aligned}
& \int_{x_{i-1}}^{x_{i}} \frac{\alpha(y)\left(1-\phi_{i}(y)\right)}{\left(x_{i}-y\right)^{1+\gamma}} d y \\
& \quad \approx \frac{\alpha\left(x_{i}\right) h^{-\gamma}}{1-\gamma}-\frac{\alpha^{\prime}\left(x_{i}\right) h^{1-\gamma}}{2-\gamma}+\frac{\alpha^{\prime \prime}\left(x_{i}\right) h^{2-\gamma}}{2(3-\gamma)}-\frac{\alpha^{\prime \prime \prime}\left(x_{i}\right) h^{3-\gamma}}{6(4-\gamma)} .
\end{aligned}
$$

$\{$ Mati:e7\}

We combine equations (16)-(22) to approximate the diagonal entries of the stiffness matrix as follows

$$
A_{i, i} \approx 4 \alpha\left(x_{i}\right)\left(\frac{h^{-\gamma}-\delta^{-\gamma}}{\gamma}+\frac{h^{-\gamma}}{1-\gamma}\right)+\alpha^{\prime \prime}\left(x_{i}\right)\left(\frac{\delta^{2-\gamma}-h^{2-\gamma}}{2-\gamma}+\frac{h^{2-\gamma}}{3-\gamma}\right) .
$$

\subsection{Evaluation of the off-diagonal entries of the stiffness matrix}

We now turn to the off-diagonal entries of the matrix $\mathbf{A}$ given in (8)

$$
A_{i, j}=-\alpha\left(x_{i}\right) \int_{x_{i}-\delta}^{x_{i}+\delta} \frac{\phi_{j}(y)}{\left|x_{i}-y\right|^{1+\gamma}} d y-\int_{x_{i}-\delta}^{x_{i}+\delta} \frac{\alpha(y) \phi_{j}(y)}{\left|x_{i}-y\right|^{1+\gamma}} d y, \quad j \neq i .
$$

$\{$ Matij:e1\}

Since the support of $\phi_{j}$ is the interval $\left[x_{j-1}, x_{j+1}\right]$, it is clear that

$$
A_{i, j}=0, \quad|i-j| \geq K+2 .
$$

In other words, the stiffness matrix $\mathbf{A}$ is a dense banded matrix with the band width of $K+1$ which is $O(N)$ asymptotically.

We need only to evaluate the off-diagonal entries $A_{i, j}$ for $1 \leq|i-j| \leq$ $K+1$ in different cases. As in Section 4.1, one of the key issues is how to approximate the coefficient $\alpha(y)$ inside the integration in the second term on the right-hand side of (24). We choose to approximate $\alpha(y)$ on each cell $\left[x_{j-1}, x_{j}\right]$ by $\alpha\left(x_{j-1 / 2}\right)$.

Case 1: $i+1 \leq j \leq i+K-1$. Then the support of $\phi_{j}$ lies inside $\left[x_{i}, x_{i+K}\right]$.

$$
\begin{aligned}
A_{i, j}= & -\alpha\left(x_{i}\right) \int_{x_{j-1}}^{x_{j+1}} \frac{\phi_{j}(y)}{\left(y-x_{i}\right)^{1+\gamma}} d y-\int_{x_{j-1}}^{x_{j+1}} \frac{\alpha(y) \phi_{j}(y)}{\left(y-x_{i}\right)^{1+\gamma}} d y \\
\approx & -\alpha\left(x_{j-\frac{1}{2}}\right) \int_{x_{j-1}}^{x_{j}} \frac{y-x_{j-1}}{h\left(y-x_{i}\right)^{1+\gamma}} d y-\alpha\left(x_{j+\frac{1}{2}}\right) \int_{x_{j}}^{x_{j+1}} \frac{x_{j+1}-y}{h\left(y-x_{i}\right)^{1+\gamma}} d y \\
& -\alpha\left(x_{i}\right)\left[\int_{x_{j-1}}^{x_{j}} \frac{y-x_{j-1}}{h\left(y-x_{i}\right)^{1+\gamma}}+\int_{x_{j}}^{x_{j+1}} \frac{x_{j+1}-y}{h\left(y-x_{i}\right)^{1+\gamma}} d y\right] \\
= & -\alpha\left(x_{j-\frac{1}{2}}\right) l_{j-i}-\alpha\left(x_{j+\frac{1}{2}}\right) r_{j-i}-\alpha\left(x_{i}\right)\left(l_{j-i}+r_{j-i}\right),
\end{aligned}
$$


where $l_{k}$ and $r_{k}$, with $k:=j-i=1,2, \ldots, K-1$, are defined by

$$
\begin{aligned}
& l_{k}:= \begin{cases}\frac{h^{-\gamma}}{\gamma}(1-k)\left[(k-1)^{-\gamma}-k^{-\gamma}\right]+\frac{h^{-\gamma}}{1-\gamma}\left[k^{1-\gamma}-(k-1)^{1-\gamma}\right], & \gamma \neq 0, \\
1+(1-k) \ln \left(\frac{k}{k-1}\right), & \gamma=0,\end{cases} \\
& r_{k}:= \begin{cases}\frac{h^{-\gamma}}{\gamma}(k+1)\left[k^{-\gamma}-(k+1)^{-\gamma}\right]+\frac{h^{-\gamma}}{1-\gamma}\left[k^{1-\gamma}-(k+1)^{1-\gamma}\right], & \gamma \neq 0, \\
(k+1) \ln \left(\frac{k+1}{k}\right), & \gamma=0 .\end{cases}
\end{aligned}
$$

Case 2: $i-K+1 \leq j \leq i-1$. Hence,

$$
\begin{aligned}
A_{i, j} & =-\alpha\left(x_{i}\right) \int_{x_{j-1}}^{x_{j+1}} \frac{\phi_{j}(y)}{\left(x_{i}-y\right)^{1+\gamma}} d y-\int_{x_{j-1}}^{x_{j+1}} \frac{\alpha(y) \phi_{j}(y)}{\left(x_{i}-y\right)^{1+\gamma}} d y \\
& \approx-\alpha\left(x_{j+\frac{1}{2}}\right) l_{i-j}-\alpha\left(x_{j-\frac{1}{2}}\right) r_{i-j}-\alpha\left(x_{i}\right)\left(l_{i-j}+r_{i-j}\right) .
\end{aligned}
$$

Case 3: $j=i+K \cdot \operatorname{supp}\left\{\phi_{i+K}\right\}=\left[x_{i+K-1}, x_{i+K+1}\right] \not \subset\left[x_{i}, x_{i}+\delta\right]$, in general. We evaluate $A_{i, i+K}$ accordingly

$$
\begin{aligned}
A_{i, i+K}= & -\alpha\left(x_{i}\right) \int_{x_{i+K-1}}^{x_{i}+\delta} \frac{\phi_{j}(y)}{\left(y-x_{i}\right)^{1+\gamma}} d y-\int_{x_{i+K-1}}^{x_{i}+\delta} \frac{\alpha(y) \phi_{j}(y)}{\left(y-x_{i}\right)^{1+\gamma}} d y \\
\approx & -\alpha\left(x_{i}\right)\left[\int_{x_{i+K-1}}^{x_{i+K}} \frac{y-x_{i+K-1}}{h\left(y-x_{i}\right)^{1+\gamma}} d y+\int_{x_{i+K}}^{x_{i}+\delta} \frac{x_{i+K+1}-y}{h\left(y-x_{i}\right)^{1+\gamma}} d y\right] \\
& -\alpha\left(x_{i+K-\frac{1}{2}}\right) \int_{x_{i+K-1}}^{x_{i+K}} \frac{y-x_{i+K-1}}{h\left(y-x_{i}\right)^{1+\gamma}} d y \\
& -\alpha\left(x_{i+K+\frac{1}{2}}\right) \int_{x_{i+K}}^{x_{i}+\delta} \frac{x_{i+K+1}-y}{h\left(y-x_{i}\right)^{1+\gamma}} d y \\
= & -\alpha\left(x_{i+K-\frac{1}{2}}\right) l_{K}-\alpha\left(x_{i+K+\frac{1}{2}}\right) r_{K}-\alpha\left(x_{i}\right)\left(l_{K}+r_{K}\right)
\end{aligned}
$$

where

$$
l_{K}:=\left\{\begin{array}{cc}
\frac{h^{-\gamma}}{\gamma}(1-K)\left[(K-1)^{-\gamma}-K^{-\gamma}\right] & \\
+\frac{h^{-\gamma}}{1-\gamma}\left[K^{1-\gamma}-(K-1)^{1-\gamma}\right], & \gamma \neq 0, \\
1+(1-K) \ln \left(\frac{K}{K-1}\right), & \gamma=0,
\end{array}\right.
$$




$$
r_{K}:= \begin{cases}(K+1) \frac{(K h)^{-\gamma}-\delta^{-\gamma}}{\gamma}-\frac{\delta^{1-\gamma}-(K h)^{1-\gamma}}{h(1-\gamma)}, & \gamma \neq 0, \\ (K+1) \ln \left(\frac{\delta}{K h}\right)-\frac{\delta-K h}{h}, & \gamma=0 .\end{cases}
$$

Case 4: $j=i-K$. By symmetry, we have

$$
A_{i, i-K}=-\alpha\left(x_{i-K+\frac{1}{2}}\right) l_{K}-\alpha\left(x_{i-K-\frac{1}{2}}\right) r_{K}-\alpha\left(x_{i}\right)\left(l_{K}+r_{K}\right) .
$$

Case 5: $j=i+K+1 \cdot \operatorname{supp}\left\{\phi_{i+K+1}\right\}=\left[x_{i+K}, x_{i+K+2}\right] \not \subset\left[x_{i}, x_{i}+\delta\right]$, in general. We evaluate $A_{i, i+K+1}$ accordingly.

$$
\begin{aligned}
A_{i, i+K+1} & =-\alpha\left(x_{i}\right) \int_{x_{i+K}}^{x_{i}+\delta} \frac{y-x_{i+K}}{h\left(y-x_{i}\right)^{1+\gamma}} d y-\int_{x_{i+K}}^{x_{i}+\delta} \frac{\alpha(y)\left(y-x_{i+K}\right)}{h\left(y-x_{i}\right)^{1+\gamma}} d y \\
& \approx-\left(\alpha\left(x_{i}\right)+\alpha\left(x_{i+K+\frac{1}{2}}\right)\right) \int_{x_{i+K}}^{x_{i}+\delta} \frac{y-x_{i+K}}{h\left(y-x_{i}\right)^{1+\gamma}} d y \\
& =-\alpha\left(x_{i+K+\frac{1}{2}}\right) l_{K+1}-\alpha\left(x_{i}\right) l_{K+1} .
\end{aligned}
$$

Here

$$
l_{K+1}:= \begin{cases}\frac{K \delta^{-\gamma}}{\gamma}-\frac{K^{1-\gamma} h^{-\gamma}}{\gamma}+\frac{\delta^{1-\gamma}-(K h)^{1-\gamma}}{h(1-\gamma)}, & \gamma \neq 0, \\ -K \ln \left(\frac{\delta}{K h}\right)+\frac{\delta-K h}{h}, & \gamma=0 .\end{cases}
$$

Case 6: $j=i-K-1$. By symmetry, we have

$$
\begin{aligned}
A_{i, i-K-1} & =-\alpha\left(x_{i}\right) \int_{x_{i}-\delta}^{x_{i-K}} \frac{x_{i-K}-y}{h\left(x_{i}-y\right)^{1+\gamma}} d y-\int_{x_{i}-\delta}^{x_{i-K}} \frac{\alpha(y)\left(x_{i-K}-y\right)}{h\left(x_{i}-y\right)^{1+\gamma}} d y \\
& \approx-\left(\alpha\left(x_{i}\right)+\alpha\left(x_{i-K-\frac{1}{2}}\right)\right) \int_{x_{i}-\delta}^{x_{i-K}} \frac{x_{i-K}-y}{h\left(x_{i}-y\right)^{1+\gamma}} d y \\
& =-\alpha\left(x_{i-K-\frac{1}{2}}\right) l_{K+1}-\alpha\left(x_{i}\right) l_{K+1} .
\end{aligned}
$$

\section{Structure of the stiffness matrix}

We are now in a position to prove the structure of the stiffness matrix $\mathbf{A}$. 
Theorem 1. The stiffness matrix $\mathbf{A}$ can be decomposed as

$$
\begin{aligned}
\mathbf{A}= & d_{1}^{h, \delta} \operatorname{diag}(\boldsymbol{\alpha})+d_{2}^{h, \delta} \operatorname{diag}\left(\boldsymbol{\alpha}^{\prime \prime}\right)+\mathbf{T} \operatorname{diag}\left(\boldsymbol{\alpha}^{-}\right) \\
& +\mathbf{T}^{T} \operatorname{diag}\left(\boldsymbol{\alpha}^{+}\right)+\operatorname{diag}(\boldsymbol{\alpha})\left(\mathbf{T}+\mathbf{T}^{T}\right) .
\end{aligned}
$$

$\{$ Structure: $\mathrm{e} 1\}$

Here $\mathbf{T}:=\mathbf{L}^{T}+\mathbf{R}$, and

$$
\begin{array}{ll}
\boldsymbol{\alpha}:=\left[\alpha\left(x_{1}\right), \ldots, \alpha\left(x_{N-1}\right)\right]^{T}, & \boldsymbol{\alpha}^{-}:=\left[\alpha\left(x_{1 / 2}\right), \ldots, \alpha\left(x_{N-3 / 2}\right)\right]^{T}, \\
\boldsymbol{\alpha}^{\prime \prime}:=\left[\alpha^{\prime \prime}\left(x_{1}\right), \ldots, \alpha^{\prime \prime}\left(x_{N-1}\right)\right]^{T}, & \boldsymbol{\alpha}^{+}:=\left[\alpha\left(x_{3 / 2}\right), \ldots, \alpha\left(x_{N-1 / 2}\right)\right]^{T}, \\
d_{1}^{h, \delta}:=4\left(\frac{h^{-\gamma}-\delta^{-\gamma}}{\gamma}+\frac{h^{-\gamma}}{1-\gamma}\right), & d_{2}^{h, \delta}:=\left(\frac{\delta^{2-\gamma}-h^{2-\gamma}}{2-\gamma}+\frac{h^{2-\gamma}}{3-\gamma}\right),
\end{array}
$$

with

$$
\mathbf{L}:=\left(\begin{array}{cccccccccc}
0 & 0 & \ldots & \ldots & 0 & 0 & \ldots & \ldots & 0 & 0 \\
-l_{1} & 0 & 0 & \ldots & 0 & 0 & \ddots & \ddots & \ddots & 0 \\
\vdots & -l_{1} & 0 & \ldots & \ddots & 0 & \ddots & \ddots & \ddots & \vdots \\
\vdots & \vdots & \vdots & \ddots & \vdots & \vdots & \ddots & \ddots & \ddots & \vdots \\
-l_{K+1} & -l_{K} & \ddots & \ldots & 0 & 0 & \ldots & \ddots & 0 & 0 \\
0 & -l_{K+1} & -l_{K} & \ldots & -l_{1} & 0 & \ldots & \ldots & 0 & 0 \\
\vdots & \ddots & \ddots & \ddots & \vdots & \vdots & \ddots & \ddots & \vdots & \vdots \\
\vdots & \ddots & \ddots & \ddots & \ddots & \vdots & \ddots & \ddots & \vdots & \vdots \\
0 & \ddots & \ddots & \ddots & -l_{K+1} & -l_{K} & \ldots & \ldots & 0 & 0 \\
0 & 0 & \ldots & \ldots & 0 & -l_{K+1} & \ldots & \ldots & -l_{1} & 0
\end{array}\right),
$$

(34) $\{$ MatLlow 


$$
\mathbf{R}:=\left(\begin{array}{cccccccccc}
0 & 0 & \ldots & \ldots & 0 & 0 & \ldots & \ldots & 0 & 0 \\
-r_{1} & 0 & 0 & \ldots & 0 & 0 & \ddots & \ddots & \ddots & 0 \\
\vdots & -r_{1} & 0 & \ldots & \ddots & 0 & \ddots & \ddots & \ddots & \vdots \\
\vdots & \vdots & \vdots & \ddots & \vdots & \vdots & \ddots & \ddots & \ddots & \vdots \\
-r_{K} & -r_{K-1} & \ddots & \ldots & 0 & 0 & \ldots & \ddots & 0 & 0 \\
0 & -r_{K} & -r_{K-1} & \ldots & -r_{1} & 0 & \ldots & \ldots & 0 & 0 \\
\vdots & \ddots & \ddots & \ddots & \vdots & \vdots & \ddots & \ddots & \vdots & \vdots \\
\vdots & \ddots & \ddots & \ddots & \ddots & \vdots & \ddots & \ddots & \vdots & \vdots \\
0 & \ddots & \ddots & \ddots & -r_{K} & -r_{K-1} & \ldots & \ldots & 0 & 0 \\
0 & 0 & \ldots & \ldots & 0 & -r_{K} & \ldots & \ldots & -r_{1} & 0
\end{array}\right),
$$

$\{$ MatR $\}$

Proof. We observe from (23) that

$$
\operatorname{diag}(\mathbf{A})=d_{1}^{h, \delta} \operatorname{diag}(\boldsymbol{\alpha})+d_{2}^{h, \delta} \operatorname{diag}\left(\boldsymbol{\alpha}^{\prime \prime}\right) .
$$

$\{$ Structure:e3a\}

We now look at the decomposition of the off-diagonal entries of $\mathbf{A}$, i.e., the entries of $\mathbf{A}-\operatorname{diag}(\mathbf{A})$. By symmetry, we need only consider the decomposition of the upper triangular part of $\mathbf{A}-\operatorname{diag}(\mathbf{A})$ (with zero entries in the diagonal and lower triangular part of the matrix). This corresponds to cases 1,3 , and 5 in Section 4.2 .

The first terms on the right-hand sides of (26), (28), and (30) can be expressed as

$$
\mathbf{L}^{T} \operatorname{diag}\left(\boldsymbol{\alpha}^{-}\right)
$$

Similarly, the second terms on the right-hand sides of (26) and (28) can be written as

$$
\mathbf{R}^{T} \operatorname{diag}\left(\boldsymbol{\alpha}^{+}\right) \text {. }
$$

$\{$ Structure:e4\}

Next, the last terms on the right-hand sides of (26), (28), and (30) can be expressed as

$$
\operatorname{diag}(\boldsymbol{\alpha})\left(\mathbf{L}^{T}+\mathbf{R}^{T}\right) .
$$

$\{$ Structure:e5\}

Finally, we observe from cases 2, 4, and 6 in Section 4.2 that the lower triangular part of $\mathbf{A}-\operatorname{diag}(\mathbf{A})$ (with zero entries in the diagonal and upper triangular part of the matrix) can be expressed as

$$
\mathbf{L} \operatorname{diag}\left(\boldsymbol{\alpha}^{+}\right)+\mathbf{R} \operatorname{diag}\left(\boldsymbol{\alpha}^{-}\right)+\operatorname{diag}(\boldsymbol{\alpha})(\mathbf{L}+\mathbf{R}) .
$$

$\{$ Structure:e6\}

We combine equations (36)-(40) to finish the proof of the theorem. 
Theorem 2. The stiffness matrix A can be stored in $O(N)$ memories. For any vector $\mathbf{v} \in \mathbb{R}^{N-1}$, the matrix-vector multiplication $\mathbf{A v}$ can be carried out in $O(N \log N)$ computations.

Proof. By the expansion (32), one has to store $\boldsymbol{\alpha}, \boldsymbol{\alpha}^{-}, \boldsymbol{\alpha}^{+}, \boldsymbol{\alpha}^{\prime \prime}$, and $\mathbf{T}$ that require $O(N)$ memories. To count the computational cost of $\mathbf{A v}$, we observe from the expansion (32) that we need only count the computational cost of $\mathbf{T} \mathbf{v}$ and $\mathbf{T}^{\mathbf{T}} \mathbf{v}$ as $\mathbf{v}$ multiplied by diagonal matrices can be carried out in $O(N)$ computations. By symmetry, we need only count the computational complexity of $\mathbf{T} \mathbf{v}$.

There is a Toeplitz matrix $\mathbf{S}$ of order $N-1[3,4,26]$

$$
\mathbf{S}:=\left(\begin{array}{cccccccccc}
0 & 0 & \ldots & \ldots & 0 & -r_{K} & \ldots & \ldots & -r_{1} & 0 \\
0 & 0 & 0 & \ldots & 0 & 0 & \ddots & \ddots & \ddots & -r_{1} \\
\vdots & 0 & 0 & \ldots & \ddots & 0 & \ddots & \ddots & \ddots & \vdots \\
\vdots & \vdots & \vdots & \ddots & \vdots & \vdots & \ddots & \ddots & \ddots & \vdots \\
-l_{K+1} & 0 & \ddots & \ldots & 0 & 0 & \ldots & \ddots & 0 & -r_{K} \\
-l_{K} & -l_{K+1} & 0 & \ldots & 0 & 0 & \ldots & \ldots & 0 & 0 \\
\vdots & \ddots & \ddots & \ddots & \vdots & \vdots & \ddots & \ddots & \vdots & \vdots \\
\vdots & \ddots & \ddots & \ddots & \ddots & \vdots & \ddots & \ddots & \vdots & \vdots \\
-l_{1} & \ddots & \ddots & \ddots & -l_{K+1} & 0 & \ldots & \ldots & 0 & 0 \\
0 & -l_{1} & \ldots & \ldots & -l_{K} & -l_{K+1} & \ldots & \ldots & 0 & 0
\end{array}\right),
$$

such that

$$
\mathbf{C}_{2 N-2}:=\left(\begin{array}{cc}
\mathbf{T} & \mathbf{S} \\
\mathbf{S} & \mathbf{T}
\end{array}\right)
$$

is a circulant matrix of order $2(N-1)$-by-2(N-1). It is well known that a circulant matrix $\mathbf{C}_{2 N-2}$ can be diagonalized by the discrete Fourier transform matrix $[3,5,10]$

$$
\mathbf{C}_{2 N-2}=\mathbf{F}_{2 N-2}^{-1} \operatorname{diag}\left(\mathbf{F}_{2 N-2} \mathbf{c}_{2 N-2}\right) \mathbf{F}_{2 N-2},
$$

where $\mathbf{c}_{2 N-2}$ is the first column of $\mathbf{C}_{2 N-2}$.

For any vector $\mathbf{v} \in \mathbb{R}^{N-1}$, we extend it by a zero vector to a vector $\mathbf{w} \in \mathbb{R}^{2 N-2}$

$$
\mathbf{w}_{2 N-2}:=\left(\begin{array}{l}
\mathbf{v} \\
\mathbf{0}
\end{array}\right)
$$


Then by (41) and (43), we have

$$
\mathbf{C}_{2 N-2} \mathbf{w}_{2 N-2}=\left(\begin{array}{cc}
\mathbf{T} & \mathbf{S} \\
\mathbf{S} & \mathbf{T}
\end{array}\right)\left(\begin{array}{l}
\mathbf{v} \\
\mathbf{0}
\end{array}\right)=\left(\begin{array}{l}
\mathbf{T} \mathbf{v} \\
\mathbf{S v}
\end{array}\right) .
$$

Thus, the matrix-vector multiplication $\mathbf{T v}$ can be obtained as the subvector consisting of the first $N-1$ components of the vector $\mathbf{C}_{2 N-2} \mathbf{w}_{2 N-2}$.

On the other hand, by (42) $\mathbf{C}_{2 N-2} \mathbf{w}_{2 N-2}$ can be computed by fast Fourier transform (FFT) and inverse fast Fourier transform (iFFT) in $O(N \log N)$ operations. Hence, $\mathbf{T v}$ can be evaluated in $O(N \log N)$ operations.

Applying Theorems 5.1 and 5.2 to any Krylov subspace iterative method yields a fast version of the method in which each Krylov subspace iteration can be evaluated in $O(N \log N)$ operations.

\section{Numerical experiments}

We carry out numerical experiments to investigate the performance of the fast collocation scheme. We choose to use a fast conjugate gradient squared (CGS) solver. That is, we will compare the performance of colloation scheme (7)-(8), which is solved with Gaussian elimination (Gauss) that has traditionally been used in the numerical simulation of nonlocal diffusion modeling, the conventional CGS solver, and the fast CGS (FCGS) solver.

In the numerical experiments, we set the data in (1) as follows: The spatial domain $(a, b)=(-1,1)$, and the variable coefficient $\alpha(x)=1+\varepsilon(1-$ $\left.x^{2}\right)$ with $\varepsilon=0.1$. We choose the true solution $u(x)=(1-x)^{2}(1+x)^{2}$ with the right-hand side source term $f(x)$ being evaluated at each collocation point $x_{i}$. All the solvers are implemented using Matlab and all the experiments are performed on a 16GB-ROM laptop.

Let $u^{h}$ be the numerical solution with mesh size $h$. We measure the $L^{2}$ errors of the different solvers for successively refined mesh sizes and fit the convergence rates

$$
\left\|u^{h}-u\right\|_{L^{2}(a, b)} \leq C_{\beta} h^{\beta} .
$$

We present the numerical results along with the CPU times of each solver and the number of iterations of the iterative solvers in a sequence of tables, for different values of $\gamma$ that quantifies the singularity of the kernel in (2).

Example 1: $\gamma=\mathbf{1 / 1 0}, \delta=1 / 32$. We present the numerical results in Table 1, from which we have the following observations: (i) All the three 
Table 1: Performance of Gauss, CGS, and the FCGS in Example 1

\begin{tabular}{|c|c|c|c|c|}
\hline & $\mathrm{h}$ & $\left\|e^{h}\right\|_{L_{2}}$ & \# of Iter. & CPU Time \\
\hline \multirow{7}{*}{ Gauss } & $2^{-8}$ & $8.183349 \times 10^{-3}$ & - & $0.23 \mathrm{~s}$ \\
\hline & $2^{-9}$ & $1.892655 \times 10^{-3}$ & - & $1.47 \mathrm{~s}$ \\
\hline & $2^{-10}$ & $4.174929 \times 10^{-4}$ & - & $12.11 \mathrm{~s}$ \\
\hline & $2^{-11}$ & $9.621059 \times 10^{-5}$ & - & $1 \mathrm{~m} 58 \mathrm{~s}$ \\
\hline & $2^{-12}$ & $2.700502 \times 10^{-5}$ & - & $16 \mathrm{~m} 54 \mathrm{~s}$ \\
\hline & $2^{-13}$ & $8.679118 \times 10^{-6}$ & - & $2 \mathrm{~h} 54 \mathrm{~m}$ \\
\hline & $2^{-14}$ & $2.789573 \times 10^{-6}$ & - & $1 \mathrm{~d} 5 \mathrm{~h}$ \\
\hline \multirow{7}{*}{ CGS } & $2^{-8}$ & $8.183349 \times 10^{-3}$ & 496 & $0.35 \mathrm{~s}$ \\
\hline & $2^{-9}$ & $1.892655 \times 10^{-3}$ & 500 & $1.53 \mathrm{~s}$ \\
\hline & $2^{-10}$ & $4.174929 \times 10^{-4}$ & 549 & $8.72 \mathrm{~s}$ \\
\hline & $2^{-11}$ & $9.621059 \times 10^{-5}$ & 527 & $22.9 \mathrm{~s}$ \\
\hline & $2^{-12}$ & $2.700502 \times 10^{-5}$ & 495 & $1 \mathrm{~m} 2 \mathrm{~s}$ \\
\hline & $2^{-13}$ & $8.679118 \times 10^{-6}$ & 527 & $5 \mathrm{~m} 27 \mathrm{~s}$ \\
\hline & $2^{-14}$ & $2.789573 \times 10^{-6}$ & 570 & $21 \mathrm{~m} 21 \mathrm{~s}$ \\
\hline \multirow{8}{*}{ FCGS } & $2^{-8}$ & $8.183349 \times 10^{-3}$ & 496 & $1.38 \mathrm{~s}$ \\
\hline & $2^{-9}$ & $1.892655 \times 10^{-3}$ & 500 & $1.39 \mathrm{~s}$ \\
\hline & $2^{-10}$ & $4.174929 \times 10^{-4}$ & 549 & $3.11 \mathrm{~s}$ \\
\hline & $2^{-11}$ & $9.621059 \times 10^{-5}$ & 527 & $6.02 \mathrm{~s}$ \\
\hline & $2^{-12}$ & $2.700502 \times 10^{-5}$ & 495 & $11.4 \mathrm{~s}$ \\
\hline & $2^{-13}$ & $8.679118 \times 10^{-6}$ & 527 & $57 \mathrm{~s}$ \\
\hline & $2^{-14}$ & $2.789573 \times 10^{-6}$ & 570 & $1 \mathrm{~m} 1 \mathrm{~s}$ \\
\hline & & \multicolumn{3}{|c|}{$C_{\beta}=355$} \\
\hline
\end{tabular}


solvers generate identical numerical solutions. (ii) The CGS has better computational efficiency than Gauss, and the FCGS has the best performance among the three. (iii) The numerical scheme has a second-order convergence rate for mesh sizes down to $h=2^{-11}$, when the global truncation errors are already below the magnitude of $10^{-4}$. However, for further refined mesh sizes $h=2^{-12}, 2^{-13}, 2^{-14}$, the convergence rate of the numerical schemes reduces to about one and half orders. We feel that the deterioration of the convergence rate may be a result of the numerical integration of singular integrals (e.g., in the calculation of the righ-hand side source term $f\left(x_{i}\right)$ ). Since the objective of this paper is to study fast solution techniques, we do not push further in this direction.

Table 2: Performance of Gauss, CGS, and the FCGS in Example 2

\begin{tabular}{|c|c|c|c|c|}
\hline & $\mathrm{h}$ & $\left\|e^{h}\right\|_{L_{2}}$ & \# of Iter. & CPU Time \\
\hline \multirow{5}{*}{ Gauss } & $2^{-8}$ & $1.805186 \times 10^{-2}$ & - & $0.22 \mathrm{~s}$ \\
\hline & $2^{-9}$ & $5.457536 \times 10^{-3}$ & - & $1.37 \mathrm{~s}$ \\
\hline & $2^{-10}$ & $1.496009 \times 10^{-3}$ & - & $12.12 \mathrm{~s}$ \\
\hline & $2^{-11}$ & $3.957454 \times 10^{-4}$ & - & $2 \mathrm{~m} 18 \mathrm{~s}$ \\
\hline & $2^{-12}$ & $1.057274 \times 10^{-4}$ & - & $16 \mathrm{~m} 57 \mathrm{~s}$ \\
\hline \multirow{5}{*}{ CGS } & $2^{-8}$ & $1.805186 \times 10^{-2}$ & 758 & $0.68 \mathrm{~s}$ \\
\hline & $2^{-9}$ & $5.457536 \times 10^{-3}$ & 896 & $2.57 \mathrm{~s}$ \\
\hline & $2^{-10}$ & $1.496009 \times 10^{-3}$ & 1011 & $14.6 \mathrm{~s}$ \\
\hline & $2^{-11}$ & $3.957454 \times 10^{-4}$ & 1139 & $51.98 \mathrm{~s}$ \\
\hline & $2^{-12}$ & $1.057274 \times 10^{-4}$ & 1358 & $3 \mathrm{~m} 58 \mathrm{~s}$ \\
\hline \multirow{6}{*}{ FCGS } & $2^{-8}$ & $1.805186 \times 10^{-2}$ & 758 & $1.19 \mathrm{~s}$ \\
\hline & $2^{-9}$ & $5.457536 \times 10^{-3}$ & 896 & $3.00 \mathrm{~s}$ \\
\hline & $2^{-10}$ & $1.496009 \times 10^{-3}$ & 1011 & $5.35 \mathrm{~s}$ \\
\hline & $2^{-11}$ & $3.957454 \times 10^{-4}$ & 1139 & $12.02 \mathrm{~s}$ \\
\hline & $2^{-12}$ & $1.057274 \times 10^{-4}$ & 1358 & $30.07 \mathrm{~s}$ \\
\hline & & \multicolumn{3}{|c|}{$C_{\beta}=578$} \\
\hline
\end{tabular}

Example 2: $\gamma=\mathbf{1 / 2}, \delta=1 / 32$. Namely, the singularity of the kernel gets stronger. We present the numerical results in Table 2. We have similar observations to those for Example 1 except for the following: (i) The magnitude of the global truncation errors of the numerical schemes gets bigger. (ii) The convergence rates cannot reach second-order any longer, although still 
quite close. Both (i) and (ii) coincide with our previous speculation that the singularity of the kernel plays an important rule in the deterioration of the global truncation errors and the convergence rates of the numerical schemes. (iii) Most notably, the number of iterations of the CGS and the fast CGS gets more than doubled. The CPU time of CGS is almost quadrapled, while that of the FCGS is more than two and half times of the CPU time for the case $\gamma=1 / 10$. In contrast, the CPU time of Gauss remains unchanged an anticiplated, since Gauss elimination has no iterations involved. Of course, Gauss still consumes significantly more CPU time than the FCGS.

Table 3: Performance of Gauss, CGS, and the FCGS in Example 3

\begin{tabular}{|c|c|c|c|c|}
\hline & $\mathrm{h}$ & $\left\|e^{h}\right\|_{L_{2}}$ & \# of Iter. & CPU Time \\
\hline \multirow{5}{*}{ Gauss } & $2^{-8}$ & $3.457841 \times 10^{-2}$ & - & $0.22 \mathrm{~s}$ \\
\hline & $2^{-9}$ & $1.384261 \times 10^{-2}$ & - & $1.42 \mathrm{~s}$ \\
\hline & $2^{-10}$ & $4.828425 \times 10^{-3}$ & - & $12.31 \mathrm{~s}$ \\
\hline & $2^{-11}$ & $1.553268 \times 10^{-3}$ & - & $2 \mathrm{~m} 20 \mathrm{~s}$ \\
\hline & $2^{-12}$ & $4.812208 \times 10^{-4}$ & - & $16 \mathrm{~m} 59 \mathrm{~s}$ \\
\hline \multirow{5}{*}{ CGS } & $2^{-8}$ & $3.457841 \times 10^{-2}$ & 1005 & $0.74 \mathrm{~s}$ \\
\hline & $2^{-9}$ & $1.384261 \times 10^{-2}$ & 1464 & $4.07 \mathrm{~s}$ \\
\hline & $2^{-10}$ & $4.828425 \times 10^{-3}$ & 1836 & $29.75 \mathrm{~s}$ \\
\hline & $2^{-11}$ & $1.553268 \times 10^{-3}$ & 2362 & $1 \mathrm{~m} 43 \mathrm{~s}$ \\
\hline & $2^{-12}$ & $4.812208 \times 10^{-4}$ & 2954 & $6 \mathrm{~m} 14 \mathrm{~s}$ \\
\hline \multirow{6}{*}{ FCGS } & $2^{-8}$ & $3.457841 \times 10^{-2}$ & 1005 & $1.82 \mathrm{~s}$ \\
\hline & $2^{-9}$ & $1.384261 \times 10^{-2}$ & 1464 & $4.94 \mathrm{~s}$ \\
\hline & $2^{-10}$ & $4.828425 \times 10^{-3}$ & 1836 & $9.57 \mathrm{~s}$ \\
\hline & $2^{-11}$ & $1.553268 \times 10^{-3}$ & 2362 & $24.97 \mathrm{~s}$ \\
\hline & $2^{-12}$ & $4.812208 \times 10^{-4}$ & 2954 & $1 \mathrm{~m} 6 \mathrm{~s}$ \\
\hline & & \multicolumn{3}{|c|}{$C_{\beta}=204 \quad \beta=1.54$} \\
\hline
\end{tabular}

Example 3: $\gamma=3 / 4, \delta=1 / 32$. The singularity of the kernel gets even stronger. We present the numerical results in Table 3. We have similar observations to those for Example 2 except for the following: (i) The magnitude of the global truncation errors of the numerical schemes increase further. (ii) The convergence rates of the numerical schemes reduce to about one and half orders. (iii) The number of iterations of the CGS and the fast CGS gets more than doubled than that for the case of $\gamma=1 / 2$ at $h=2^{-12}$. 
The CPU time of CGS and the FCGS is almost doubled. In contrast, the CPU time of Gauss remains unchanged an anticiplated.

These results suggest the development of an effective and efficient preconditioner for the fast collocation scheme, which is beyond the scope of this paper and will be carried out in the near future.

Table 4: Performance of Gauss, CGS, and the FCGS in Example 4

\begin{tabular}{|c|c|c|c|c|}
\hline & $\mathrm{h}$ & $\left\|e^{h}\right\|_{L_{2}}$ & \# of Iter. & CPU Time \\
\hline \multirow{5}{*}{ Gauss } & $2^{-8}$ & $3.766216 \times 10^{-3}$ & - & $0.28 \mathrm{~s}$ \\
& $2^{-9}$ & $7.359012 \times 10^{-4}$ & - & $1.61 \mathrm{~s}$ \\
& $2^{-10}$ & $1.520926 \times 10^{-4}$ & - & $13.18 \mathrm{~s}$ \\
& $2^{-11}$ & $4.134325 \times 10^{-5}$ & - & $2 \mathrm{~m} 7 \mathrm{~s}$ \\
& $2^{-12}$ & $1.810697 \times 10^{-5}$ & - & $18 \mathrm{~m} 43 \mathrm{~s}$ \\
\hline \multirow{5}{*}{ CGS } & $2^{-8}$ & $3.766216 \times 10^{-3}$ & 322 & $0.23 \mathrm{~s}$ \\
& $2^{-9}$ & $7.359012 \times 10^{-4}$ & 291 & $0.8 \mathrm{~s}$ \\
& $2^{-10}$ & $1.520926 \times 10^{-4}$ & 252 & $4.19 \mathrm{~s}$ \\
& $2^{-11}$ & $4.134325 \times 10^{-5}$ & 254 & $11.53 \mathrm{~s}$ \\
& $2^{-12}$ & $1.810697 \times 10^{-5}$ & 259 & $34.85 \mathrm{~s}$ \\
\hline \multirow{5}{*}{ FCGS } & $2^{-8}$ & $8.183349 \times 10^{-3}$ & 322 & $0.67 \mathrm{~s}$ \\
& $2^{-9}$ & $1.892655 \times 10^{-4}$ & 291 & $1.02 \mathrm{~s}$ \\
& $2^{-10}$ & $4.174929 \times 10^{-4}$ & 252 & $1.41 \mathrm{~s}$ \\
& $2^{-11}$ & $9.621059 \times 10^{-5}$ & 254 & $2.64 \mathrm{~s}$ \\
& $2^{-12}$ & $2.700502 \times 10^{-5}$ & 259 & $5.78 \mathrm{~s}$ \\
\hline & & \multicolumn{4}{|c}{$C_{\beta}=153$} & $\beta=1.96$ \\
\hline
\end{tabular}

Example 4: $\gamma=-\mathbf{1} / \mathbf{2}, \delta=\mathbf{1 / 3 2}$. This example move towards the other direction of $\gamma$ from Example 1. In this case, the kernel is actually integrable. We present the numerical results in Table 4. We have similar observations to those for Example 1.

We end this section by investigating the behavior of the conditional numbers and eigenvalues of the stiffness matrices with respect to different values of $\gamma, \delta$ and $h$, respectively.

From Tables 5-7, we observe that the condition numbers of the stiffness matrix $\mathbf{A}$ increases as $N$ and $\gamma$ increase or $\delta$ decreases. This shows that a preconditioner should help reducing the number of iterations in any 
Table 5: The condition numbers, and maximum and minimum eigenvalues $\lambda_{\max }$ and $\lambda_{\min }$ of the stiffness matrices $\mathbf{A}$ for different values of $\gamma$.

\begin{tabular}{|c|c|c|c|c|}
\hline & $\gamma$ & Condition \# & $\lambda_{\max }$ & $\lambda_{\min }$ \\
\hline \multirow{4}{*}{$\begin{array}{l}N=2^{5} \\
\delta=1 / 4\end{array}$} & $-1 / 10$ & 84 & 11 & 0.13 \\
\hline & $1 / 10$ & 103 & 21 & 0.20 \\
\hline & $5 / 10$ & 168 & 89 & 0.53 \\
\hline & $9 / 10$ & 335 & 1104 & 3.30 \\
\hline \multirow{4}{*}{$\begin{array}{l}N=2^{6} \\
\delta=1 / 8\end{array}$} & $-1 / 10$ & 323 & 10 & 0.03 \\
\hline & $1 / 10$ & 369 & 22 & 0.06 \\
\hline & $5 / 10$ & 658 & 126 & 0.19 \\
\hline & $9 / 10$ & 1337 & 2070 & 1.50 \\
\hline \multirow{4}{*}{$\begin{array}{l}N=2^{7} \\
\delta=1 / 16\end{array}$} & $-1 / 10$ & 1266 & 9.8 & 0.01 \\
\hline & $1 / 10$ & 1560 & 24 & 0.02 \\
\hline & $5 / 10$ & 2606 & 179 & 0.07 \\
\hline & $9 / 10$ & 5340 & 3872 & 0.70 \\
\hline
\end{tabular}

Table 6: The condition numbers, and maximum and minimum eigenvalues $\lambda_{\max }$ and $\lambda_{\min }$ of the stiffness matrices $\mathbf{A}$ for different values of $\delta$.

\begin{tabular}{|l|c|c|c|c|}
\hline & $\delta$ & Condition $\#$ & $\lambda_{\max }$ & $\lambda_{\min }$ \\
\hline$N=2^{5}$ & $1 / 8$ & 222 & 8.6 & 0.04 \\
$\gamma=-1 / 10$ & $1 / 4$ & 84 & 11 & 0.13 \\
& $1 / 2$ & 29 & 14 & 0.47 \\
\hline$N=2^{6}$ & $1 / 16$ & 880 & 8.1 & 0.01 \\
$\gamma=-1 / 10$ & $1 / 8$ & 323 & 10 & 0.03 \\
& $1 / 4$ & 105 & 13 & 0.12 \\
\hline$N=2^{7}$ & $1 / 32$ & 3504 & 7.6 & 0.002 \\
$\gamma=-1 / 10$ & $1 / 16$ & 1266 & 9.8 & 0.008 \\
& $1 / 8$ & 401 & 12 & 0.03 \\
\hline
\end{tabular}


Table 7: The condition numbers, and maximum and minimum eigenvalues $\lambda_{\max }$ and $\lambda_{\min }$ of the stiffness matrices $\mathbf{A}$ for different values of $N$.

\begin{tabular}{|l|c|c|c|c|}
\hline & $N$ & Condition \# & $\lambda_{\max }$ & $\lambda_{\min }$ \\
\hline & $2^{5}$ & 29 & 14 & 0.47 \\
$\gamma=-1 / 10$ & $2^{6}$ & 35 & 16 & 0.45 \\
$\delta=1 / 2$ & $2^{7}$ & 39 & 17 & 0.45 \\
& $2^{8}$ & 43 & 19 & 0.44 \\
\hline & $2^{5}$ & 84 & 11 & 0.13 \\
$\gamma=-1 / 10$ & $2^{6}$ & 105 & 13 & 0.12 \\
$\delta=1 / 4$ & $2^{7}$ & 123 & 15 & 0.12 \\
& $2^{8}$ & 138 & 16 & 0.12 \\
\hline & $2^{5}$ & 222 & 8.6 & 0.04 \\
$\gamma=-1 / 10$ & $2^{6}$ & 323 & 10 & 0.03 \\
$\delta=1 / 8$ & $2^{7}$ & 401 & 12 & 0.03 \\
& $2^{8}$ & 463 & 14 & 0.03 \\
\hline
\end{tabular}

Krylov subspace iterative method. However, the development of such a preconditioner is not as simple as the preconditioners developed for numerical methods for fractional partial differential equations [12, 23], due to the complicated structure of the stiffness matrix $\mathbf{A}$ as proved in Theorem 1 . This will be investigated in the near future.

\section{Concluding remarks}

In this paper we consider a one-dimensional variable-coefficient nonlocal diffusion model [15], for which virtually any numerical discretization would yield a dense stiffness matrix. A conventional direct solver would require $O\left(N^{2}\right)$ memory and $O\left(N^{3}\right)$ computational work for a discretization with $N$ degrees of freedom. Furthermore, because the variable diffusivity coefficient appears inside the singular integral operator, the fast numerical methods developed for space-fractional partial differential equations $[1,22,26]$ or constant-coefficient nonlocal diffusion models [21, 24, 25] do not apply.

In this paper we develop a fast collocation method for the nonlocal diffusion model with a smooth diffusivity coefficient, by carefully handling the variable coefficients appearing inside the singular integral operator and by ex- 
ploiting the structure of the dense stiffness matrix. The resulting fast method reduces the computational work from $O\left(N^{3}\right)$ required by a commonly used direct solver to $O(N \log N)$ per iteration and the memory requirement from $O\left(N^{2}\right)$ to $O(N)$. Furthermore, the fast method reduces the computational work of assembling the stiffness matrix from $O\left(N^{2}\right)$ to $O(N)$ and is matrixfree. Namely, one does not need to store the full stiffness matrix. In this paper we carry out numerical experiments for different values of $\gamma$ and $\delta$ to observe the performance of the fast method, which show the utility of the fast method.

Although they have a very similar form in one space dimension, the nonlocal diffusion model and the peridynamic model represent very different physical processes with different properties (relatively smooth anomalous diffusion processes vs. elasticity problems with cracks and fractures) [19, 20]. For instance, peridynamic models correspond to (2) with $\gamma=0$ that have a relatively weak singularity. On the other hand, peridynamic models are used to model problems with singularities. In other words, we need to develop a fast method discretized on locally refined meshes. In other words, to extend the fast method developed for the nonlocal diffusion model to peridynamic models, we need to conduct further research. This will be investigated in the near future.

\section{Acknowledgements}

This work is supported in part by the OSD/ARO MURI Grant W911NF15-1-0562 and by the National Science Foundation under Grants DMS-1216923 and DMS-1620194. The authors would like to express their sincere thanks to the referees for their very helpful comments and suggestions, which greatly improved the quality of this paper.

[1] T.S. Basu and H. Wang, A fast second-order finite difference method for space-fractional diffusion equations, Int'l J. Numer. Anal. Modeling, 9 (2012), 658-666.

[2] D. Benson, S.W. Wheatcraft, and M.M. Meerschaert, The fractionalorder governing equation of Lévy motion. Water Resour. Res. 36 (2000), 1413-1423.

[3] R.H. Chan and X. Jin, An Introduction to Iterative Toeplitz Solvers, SIAM, Philadelphia, 2007. 
[4] R.H. Chan and M.K. Ng, Conjugate gradient methods for Toeplitz systems, SIAM Review, 38 (1996), 427-482.

[5] P.J. Davis, Circulant Matrices, Wiley-Intersciences, New York, 1979.

[6] D. del-Castillo-Negrete, B.A. Carreras, and V. E. Lynch, Fractional diffusion in plasma turbulence, Phys. Plasmas, 11 (2004), 3854.

[7] M. D'Elia, R. Lehoucq, M. Gunzburger, and Q. Du, Finite range jump processes and volume-constrained diffusion problems, Sandia National Labs SAND, 2014-2584.

[8] Q. Du, M. Gunzburger, R. Lehoucq, and K. Zhou, Analysis and approximation of nonlocal diffusion problems with volume constraints, SIAM Review, 54 (2012), 667-696.

[9] D. del-Castillo-Negrete, B.A. Carreras, and V. E. Lynch, Fractional diffusion in plasma turbulence, Phys. Plasmas, 11 (2004), 3854.

[10] R.M. Gray, Toeplitz and Circulant Matrices: A Review, Foundations and Trends in Comm 2, (2006), 155-239.

[11] M. Gunzburger, R Lehoucq, A nonlocal vector calculus with application to nonlocal boundary value problems, Multiscale Model. Simul. 8 (2010) 1581-1598.

[12] J. Jia and H. Wang, Fast finite difference methods for space-fractional diffusion equations with fractional derivative boundary conditions, $J$. Comput. Phys., 293 (2015), 359-369.

[13] M.M. Meerschaert and A. Sikorskii, Stochastic Models for Fractional Calculus. De Gruyter Studies in Mathematics, 2011.

[14] M.M. Meerschaert and C. Tadjeran, Finite difference approximations for two-sided space-fractional partial differential equations, Appl. Numer. Math., 56 (2006), 80-90.

[15] T. Mengesha and Q. Du, Nonlocal constrained value problems for a linear peridynamic Navier equation, J. Elast. 116 (2014), 27-51.

[16] R. Metzler and J. Klafter, The random walk's guide to anomalous diffusion: A fractional dynamics approach, Phys. Rep., 339 (2000), 1-77. 
[17] I. Podlubny, Fractional Differential Equations, Academic Press, 1999.

[18] S. Samko, A. Kilbas, and O. Marichev, Fractional Integrals and Derivatives: Theory and Applications, Gordon and Breach, London, 1993.

[19] S. Silling, Reformulation of elasticity theory for discontinuous and longrange forces, J. Mech. Phys. Solids, 48 (2000) 175-209.

[20] S. Silling, M. Epton, O. Wecker, J. Xu, and E. Askari, Peridynamic states and constitutive modeling, J. Elast., 88 (2007) 151-184.

[21] H. Tian, H. Wang, and W. Wang, An efficient collocation method for a nonlocal diffusion model, Int'l J. Numer. Anal. Modeling, 10 (2013), $815-825$.

[22] H. Wang and T.S. Basu, A fast finite difference method for twodimensional space-fractional diffusion equations, SIAM J. Sci. Comput., 34 (2012), A2444-A2458.

[23] H. Wang and N. Du, A superfast-preconditioned iterative method for steady-state space-fractional diffusion equations, J. Comput. Phys., 240 (2013), 49-57.

[24] H. Wang and H. Tian, A fast Galerkin method with efficient matrix assembly and storage for a peridynamic model, J. Comput. Phys., 231 (2012), 7730-7738.

[25] H. Wang and H. Tian, A fast and faithful collocation method with efficient matrix assembly for a two dimensional nonlocal diffusion model, Comput. Methods Appl. Mech. Engrg., 273C (2014), 19-36.

[26] H. Wang, K. Wang, and T. Sircar, A direct $O\left(N \log ^{2} N\right)$ finite difference method for fractional diffusion equations, J. Comput. Phys., 229 (2010), 8095-8104.

[27] K. Wang and H. Wang, A fast characteristic finite difference method for fractional advection-diffusion equations, Adv. Water Resour., 34 (2011), 810-816. 